\title{
Functional Connectivity of Insula, Basal Ganglia, and Prefrontal Executive Control Networks during Hypoglycemia in Type 1 Diabetes
}

\author{
-Nicolas R. Bolo, ${ }^{1,2 \star}$ Gail Musen, ${ }^{2,3 *}$ Donald C. Simonson, ${ }^{2,4}$ Lisa D. Nickerson, ${ }^{1,2}$ Veronica L. Flores, ${ }^{3}$ Tamar Siracusa, ${ }^{1}$ \\ (D)Brandon Hager, ${ }^{5}$ In Kyoon Lyoo, ${ }^{6}$ Perry F. Renshaw, ${ }^{1,2}$ and Alan M. Jacobson ${ }^{2,3,7}$ \\ ${ }^{1}$ Brain Imaging Center, McLean Hospital, Belmont, Massachusetts 02478, ${ }^{2}$ Harvard Medical School, Boston, Massachusetts 02115, ${ }^{3}$ Research Division, \\ Joslin Diabetes Center, Boston, Massachusetts 02215, ${ }^{4}$ Division of Endocrinology, Diabetes, and Hypertension, Brigham and Women's Hospital, Boston, \\ Massachusetts 02115, ${ }^{5}$ Department of Psychiatry, Beth Israel Deaconess Medical Center, Boston, Massachusetts 02215, ${ }^{6}$ Ewha Brain Institute and Graduate \\ School of Pharmaceutical Sciences, Ewha Womans University, Seoul 120-750, South Korea, and 7 Research Institute, Winthrop University Hospital, \\ Mineola, New York 11501
}

Human brain networks mediating interoceptive, behavioral, and cognitive aspects of glycemic control are not well studied. Using group independent component analysis with dual-regression approach of functional magnetic resonance imaging data, we examined the functional connectivity changes of large-scale resting state networks during sequential euglycemic-hypoglycemic clamp studies in patients with type 1 diabetes and nondiabetic controls and how these changes during hypoglycemia were related to symptoms of hypoglycemia awareness and to concurrent glycosylated hemoglobin (HbA1c) levels. During hypoglycemia, diabetic patients showed increased functional connectivity of the right anterior insula and the prefrontal cortex within the executive control network, which was associated with higher HbAlc. Controls showed decreased functional connectivity of the right anterior insula with the cerebellum/basal ganglia network and of temporal regions within the temporal pole network and increased functional connectivity in the default mode and sensorimotor networks. Functional connectivity reductions in the right basal ganglia were correlated with increases of self-reported hypoglycemic symptoms in controls but not in patients. Resting state networks that showed different group functional connectivity during hypoglycemia may be most sensitive to glycemic environment, and their connectivity patterns may have adapted to repeated glycemic excursions present in type 1 diabetes. Our results suggest that basal ganglia and insula mediation of interoceptive awareness during hypoglycemia is altered in type 1 diabetes. These changes could be neuroplastic adaptations to frequent hypoglycemic experiences. Functional connectivity changes in the insula and prefrontal cognitive networks could also reflect an adaptation to changes in brain metabolic pathways associated with chronic hyperglycemia.

Key words: executive control resting-state network; functional connectivity; functional magnetic resonance imaging; hypoglycemia; independent component analysis; type 1 diabetes

Significance Statement

The major factor limiting improved glucose control in type 1 diabetes is the significant increase in hypoglycemia associated with insulin treatment. Repeated exposure to hypoglycemia alters patients' ability to recognize the autonomic and neuroglycopenic symptoms associated with low plasma glucose levels. We examined brain resting state networks during the induction of hypoglycemia in diabetic and control subjects and found differences in networks involved in sensorimotor function, cognition, and interoceptive awareness that were related to chronic levels of glycemic control. These findings identify brain regions that are sensitive to variations in plasma glucose levels and may also provide a basis for understanding the mechanisms underlying the increased incidence of cognitive impairment and affective disorders seen in patients with diabetes.

\section{Introduction}

The successful homeostatic control of blood glucose levels requires interoceptive sensing of declining glycemic levels, as well as a complex behavioral response involving awareness of hypoglycemic symptoms, motivation, and nutrient intake. Brain func- tional units organized as interconnected networks mediate these neural processes. The insula integrates interoceptive, exteroceptive, and emotional awareness with cognitive control (Simmons et al., 2013). The basal ganglia are involved in learning, motivated behaviors, and habit formation (Yin and Knowlton, 2006). The 
prefrontal cortex is critical for cognition and executive control. However, little is known about the coordinated functioning of these brain regions as networks to mediate the cognitive and behavioral aspects related to changes in blood glucose levels.

In this study, we investigated large-scale brain resting state network (RSN) functional connectivity (FC) changes during the gradual induction of hypoglycemia in patients with type 1 diabetes mellitus (T1DM) using blood oxygen level-dependent (BOLD) functional magnetic resonance imaging (fMRI). We analyzed whole-brain fMRI time series using independent component analysis (ICA) to detect RSNs (Beckmann et al., 2005; Fox and Raichle, 2007). A better understanding of how FC changes during hypoglycemia in T1DM and of the differences between patients with T1DM and healthy subjects may help identify therapeutic targets for reducing the effect of hypoglycemia on the brain.

T1DM is caused by autoimmune destruction of pancreatic $\beta$ cells, resulting in nearly complete deficiency of insulin secretion. Patients must take exogenous insulin by subcutaneous injection, typically two to four separate injections per day or by a continuous infusion pump, to maintain control of plasma glucose levels. Because of the inevitable mismatches that occur between the administered insulin dose and daily variations in food intake and physical activity, periods of excessive hyperglycemia or hypoglycemia occur frequently. Because the brain relies almost exclusively on plasma glucose as an energy source, the effect of hypoglycemia on brain function is an area of intense physiological and clinical interest. Our goal was to study the response to a gradual glucose decline experienced typically by an individual with T1DM during daily life. Glucose also provides precursors to excitatory glutamate and inhibitory GABA neurotransmitters, shown to predict large-scale network connectivity (Kapogiannis et al., 2013). We showed previously that increased hemoglobin Alc (HbAlc) is associated with increased brain glucose and glutamate-glutamine-GABA, with decreased memory and executive function and increased depressive symptom scores, suggesting that perturbed neurotransmitter metabolism related to persistent hyperglycemia may affect brain function (Lyoo et al., 2009). Thus, glycemic excursions could affect basal neurotransmitter metabolism and brain network connectivity and function. Plasma insulin may also alter regional brain glucose metabolism (Schulingkamp et al., 2000; Bingham et al., 2002) and affect brain function (Craft and Watson, 2004). By using the hyperinsulinemic clamp method to induce euglycemia and hy-

Received Jan. 24, 2015; revised June 12, 2015; accepted June 28, 2015.

Author contributions: N.R.B., G.M., D.C.S., and A.M.J. designed research; N.R.B., G.M., D.C.S., V.L.F., T.S., I.K.L., P.F.R., and A.M.J. performed research; N.R.B., G.M., D.C.S., L.D.N., V.L.F., T.S., B.H., I.K.L., P.F.R., and A.M.J. analyzed data; N.R.B., G.M., D.C.S., L.D.N., V.L.F., T.S., B.H., I.K.L., P.F.R., and A.M.J. wrote the paper.

This work was supported in part by National Institutes of Health/National Institute of Diabetes and Digestive and Kidney Diseases Grants R01-DK073843, DK-60754, DK-62218-01A1, and P30-DK-36836 (Joslin Diabetes and Endocrinology Research (enter) and the Herbert Graetz Fund. We thank Judi Lauerman (Brigham and Women's Hospital, Boston, MA) and Karen Branch (Massachusetts General Hospital, Boston, MA) for their expert nursing assistance. Parts of this article were presented at the First Scientific Sessions of the American Diabetes Association Research Symposium: Diabetes and the Brain, Alexandria, VA, October 28 -30,2011, and at the 72nd Scientific Sessions of the American Diabetes Association, Philadelphia, PA, June 8-12, 2012.

P.F.R. is a second-tier consultant to Ridge Diagnostics and Kyowa Hakko Kirin. The other authors declare no competing financial interests.

${ }^{*}$ N.R.B. and G.M. contributed equally to this work.

Correspondence should be addressed to either of the following: Dr. Gail Musen, Research Division, Joslin Diabetes Center, 1 Joslin Place, Boston, MA 02215, E-mail: gail.musen@joslin.harvard.edu; or Dr. Nicolas R. Bolo at his present address: Department of Psychiatry, Beth Israel Deaconess Medical Center, 330 Brookline Avenue, Boston, MA 02215, E-mail:nbolo@bidmc.harvard.edu.

P. F. Renshaw's present address: Department of Psychiatry, University of Utah, Salt Lake City, UT 84112

DOI:10.1523/JNEUROSCI.0319-15.2015

Copyright $\odot 2015$ the authors $\quad 0270-6474 / 15 / 3511013-12 \$ 15.00 / 0$
Table 1. Demographics and clinical characteristics of subjects

\begin{tabular}{lcc}
\hline Characteristic & Type 1 diabetes $(n=16)$ & Controls $(n=10)$ \\
\hline HbA1c (\%) & $7.2 \pm 0.9[5.7-9.0]^{* * * *}$ & $5.1 \pm 0.3[4.4-5.4]$ \\
HbA1c (mmol/mol) & $55 \pm 7[39-75]^{* * *}$ & $34 \pm 2[25-36]$ \\
Fasting plasma glucose (mmol/L) & $6.3 \pm 1.6[4.1-9.2]^{*}$ & $5.1 \pm 0.2[4.8-5.5]$ \\
Age (years) & $30 \pm 8[19-46]$ & $31 \pm 11[19-49]$ \\
Female & 6 & 2 \\
Diabetes duration (years) & $16 \pm 6[5-27]$ & - \\
Fasting insulin $(\mu \mathrm{U} / \mathrm{ml})$ & $7.6 \pm 6.2[1.0-17.9]$ & $7.9 \pm 6.1[1.9-17.2]$ \\
Body mass index $\left(\mathrm{kg} / \mathrm{m}^{2}\right)$ & $24 \pm 3[21-31]$ & $24 \pm 2[21-27]$ \\
Systolic blood pressure $(\mathrm{mmHg})$ & $123 \pm 5[100-175]$ & $118 \pm 5[90-134]$ \\
Diastolic blood pressure $(\mathrm{mmHg})$ & $73 \pm 2[58-88]$ & $75 \pm 3[60-90]$ \\
\hline
\end{tabular}

Data are presented as mean \pm SD [range]; $n$ is number of subjects in group. Group comparisons: ${ }^{*} p<0.05$, ${ }^{* * *} p<0.001$.

poglycemia while maintaining plasma insulin at the same level in both groups during fMRI, we investigated glucose effects independently of insulin effects.

Based on our findings of altered task activation in a working memory-related network during hypoglycemia (Bolo et al., 2011), we hypothesized that FC changes during hypoglycemia in cognitive RSNs of patients with T1DM would differ from those of nondiabetic controls. We further hypothesized that connectivity changes within RSNs would be associated with changes in participant's perception of hypoglycemia-related neuroglycopenic and autonomic symptoms.

\section{Materials and Methods}

Subjects. The study sample consisted of 16 patients with T1DM (six females and 10 males) and 10 age-matched healthy nondiabetic controls (two females and eight males). Some data from 10 patients with T1DM and seven controls were included in previous publications (Musen et al., 2008; Bolo et al., 2011). Demographic and clinical characteristics of the subjects are presented in Table 1.

Patients with T1DM had a clinical history of sudden onset of significant hyperglycemia in a previously healthy young individual and were dependent on insulin to control hyperglycemia from the time of their initial presentation. All were currently treated with multiple daily insulin injections or a continuous subcutaneous insulin infusion pump. Diabetic and control subjects were excluded if they had the following: (1) clinically significant cardiovascular, neurologic, or psychiatric disease or malignancy; (2) drug or alcohol abuse; or (3) any contraindications to MRI, such as metallic implants, pregnancy, or claustrophobia. Diabetic subjects were also excluded if they had clinical evidence of autonomic or peripheral neuropathy, diabetic nephropathy, albumin excretion rates $>$ $300 \mathrm{mg} / \mathrm{d}$, or evidence of proliferative retinopathy by physical exam, review of medical records, or self-report. Subjects on any antihypertensive medication or with a history of hypertension were also excluded. Only right-handed subjects were included in the study.

The study protocol was approved by the Institutional Review Boards at Joslin Diabetes Center, where subjects were recruited and screened, and McLean Hospital, where the MRI studies were performed. All subjects gave informed voluntary written consent before participating.

Experimental protocol. The experimental protocol has been described previously (Musen et al., 2008; Bolo et al., 2011) and briefly reviewed below. All studies were performed in the morning after an overnight fast. Patients with T1DM had a continuous glucose monitor (CGM System Gold; Medtronic) inserted on the day before the study. If the continuous glucose monitor showed glucose $<3.3 \mathrm{mmol} / \mathrm{L}$, the study was postponed to a later date. Patients were instructed that the goal was to achieve a fasting glucose of $5.0-6.7 \mathrm{mmol} / \mathrm{L}$ on the morning of the study. The study diabetologist (D.C.S.) was in contact with the patients throughout this time to answer questions and assist with insulin dose managements. The experiment used the insulin clamp technique with four successive time periods corresponding to different plasma glucose levels: (1) baseline (30 min); (2) euglycemic clamp (40 min, target glucose of $5.0 \mathrm{mmol} /$ $\mathrm{L}$ ); (3) declining glycemia (40 min, plasma glucose reduced from 5.0 to 


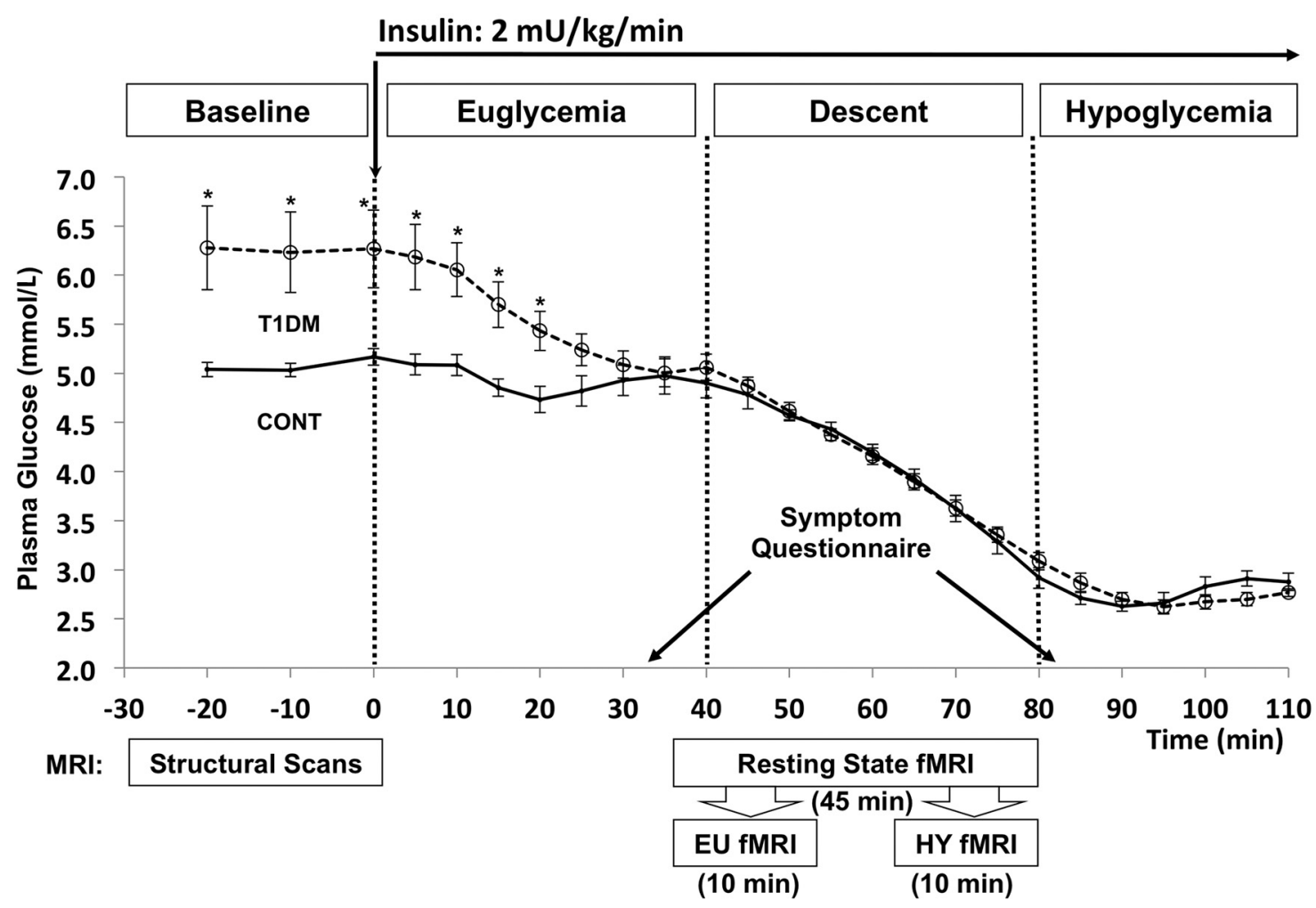

Figure 1. Experimental protocol: plasma glucose levels. The experiment used the insulin clamp technique with four successive time periods corresponding to different plasma glucose levels: (1) basal (30 min); (2) euglycemic clamp ( $40 \mathrm{~min}$, target glucose of $5.0 \mathrm{mmol} / \mathrm{L}$ ); (3) declining glycemia (40 min, plasma glucose reduced from 5.0 to $2.8 \mathrm{mmol} / \mathrm{L}$ ); and (4) hypoglycemic clamp (30 min, target glucose of $2.8 \mathrm{mmol} / \mathrm{L}$ ). Anatomical MRI was performed during baseline, and fMRI was performed during the last 5 min of the euglycemic period and the 40 min of the declining glucose period. For dual regression analysis comparing conditions, the first $10 \mathrm{~min}$ and the last $10 \mathrm{~min}$ were extracted from the full $45 \mathrm{~min}$ time series of the resting state fMRI to create the euglycemic (EU fMRI) and hypoglycemic (HY fMRI) periods, respectively. Plasma glucose levels: dashed line and open circles, T1DM; solid line and filled circles, nondiabetic control (CONT). ${ }^{*} p<0.05$ between groups.

$2.8 \mathrm{mmol} / \mathrm{L}$ ); and (4) hypoglycemic clamp (30 min, target glucose of 2.8 $\mathrm{mmol} / \mathrm{L})$. The MRI scanning began between 9:00 A.M. and 9:30 A.M. for all subjects. Anatomical MRI was performed during baseline, and fMRI was performed continuously during the last $5 \mathrm{~min}$ of the euglycemic period and the $40 \mathrm{~min}$ of the declining glucose period (total of $45 \mathrm{~min}$; Fig. 1).

Insulin clamp technique. An intravenous catheter was inserted into an antecubital vein for the administration of insulin and glucose, and a second catheter was inserted into a distal forearm or hand vein for the withdrawal of blood samples. A heated gel pack was used to warm the hand to arterialize the venous blood. After the baseline period, regular human insulin was infused at $12 \mathrm{pmol} \cdot \mathrm{kg}^{-1} \cdot \mathrm{min}^{-1}(2$ $\mathrm{mU} \cdot \mathrm{kg}^{-1} \cdot \mathrm{min}^{-1}$ ) for $110 \mathrm{~min}$ (Fig. 1). Plasma glucose was maintained at the desired level by infusion of $20 \%$ dextrose using a negative-feedback algorithm. After the euglycemic clamp period (40 min), the glucose infusion rate was reduced to allow the plasma glucose level to decline by 2.2 $\mathrm{mmol} / \mathrm{L}$ (from 5.0 to $2.8 \mathrm{mmol} / \mathrm{L}$ ) over the next $40 \mathrm{~min}$, followed by the 30 min hypoglycemic clamp period (Fig. 1). During the entire clamp protocol, glucose levels were measured every $5 \mathrm{~min}$, and counterregulatory hormones (epinephrine, cortisol, growth hormone, and glucagon) were measured every $10 \mathrm{~min}$. At the end of the protocol, the insulin infusion was discontinued, euglycemia was restored, and the subjects were given a meal.

Analytical methods. Plasma glucose was measured by the glucose oxidase method. Cortisol and glucagon were measured by radioimmunoassay. Epinephrine, growth hormone, and insulin were measured by ELISA. Statistical comparisons between groups were made using paired or unpaired Student's $t$ test, as appropriate, and ANOVA with repeated measures over time. Counter-regulatory hormones were analyzed using a longitudinal linear mixed-effects model adjusting for baseline. Prespecified contrasts between groups at each time point were performed.

Assessment of symptoms associated with hypoglycemia. On the day of the study, we administered a questionnaire (Clarke et al., 1995) to assess subjective and objective measures of hypoglycemia awareness. Patients were asked to answer questions related to the presence or absence of hypoglycemia, the blood glucose level at which symptoms occur, and the ability to sense symptoms. All subjects also responded to a hypoglycemic symptom questionnaire just before the 45 min fMRI run (during the euglycemic clamp) and just after the end of the fMRI run (during the hypoglycemic clamp; Fig. 1). The hypoglycemia symptom questionnaire comprised nine symptoms: four that were predominantly autonomic (trembling, sweating, pounding heart, fast pulse) and five that were predominantly neuroglycopenic (lightheaded, difficulty concentrating, feeling uncoordinated, feeling confused, feeling weak). Symptoms were presented on a backlit screen, visualized from within the magnet bore by a mirror mounted on the head coil, using Presentation software (Neurobehavioral Systems). Responses were made by moving a cursor on the screen to the number that corresponded to the intensity of each symptom using a magnetic resonance-compatible hand-held four-button fiberoptic response pad (fORP; Current Designs). Subjects were asked to rate each symptom on a Likert scale from one (feeling the symptom "not at all") to seven (feeling the symptom "a lot"). The autonomic and neuroglycopenic symptom scores, respectively, were determined as the sums of the scores for the individual symptoms.

MRI and fMRI data acquisition. Acquisition of MRI and fMRI data was performed using a 3.0 tesla Trio whole body scanner (Siemens), with a circularly polarized birdcage radio frequency head coil tuned to the proton frequency. Global field uniformity was adjusted at the beginning of each scanning session using Siemens automated shimming routines. A three-dimensional (3D) T1-weighted anatomical image was acquired using a magnetization-prepared rapid-acquisition gradient-echo sequence (TR, $2100 \mathrm{~ms}$; TE, $2.74 \mathrm{~ms}$; spatial resolution, $1 \times 1 \times 1.3 \mathrm{~mm}^{3}$; matrix size, $256 \times 256 \times 128)$ and used for functional image registration. BOLD fMRI images covering the whole brain were acquired in the coronal plane using an echo planar imaging sequence (EPI: TR, $6000 \mathrm{~ms}$; TE, $30 \mathrm{~ms} ; 41$ coronal slices; slice thickness, $4 \mathrm{~mm}$; field of view, $200 \times 200 \mathrm{~mm}^{2}$; 
matrix size, $64 \times 64$ ) with an in-plane resolution of $3.1 \times 3.1 \mathrm{~mm}^{2}$. A total of 450 EPI multislice volumes were acquired to cover the $45 \mathrm{~min}$ period composed of an initial $5 \mathrm{~min}$ of euglycemia, followed by $40 \mathrm{~min}$ of declining glycemia (Fig. 1). One multislice volume covering the whole brain was acquired every $6 \mathrm{~s}$. Our relatively long TR of $6 \mathrm{~s}$ is not ideal but was a tradeoff in balancing other study-related issues. We were interested in detecting the low-frequency spontaneous oscillations in BOLD signals $(\sim 0.01-0.08 \mathrm{~Hz}$, i.e., one cycle approximately every $12-100 \mathrm{~s})$, for which long TR has less impact compared with detecting more rapid task activation-related BOLD changes. We mitigated as much as possible the effects of long TR by using the full 45 min time course in the group ICA to include as many oscillations as possible and by using $10 \mathrm{~min}$ of fMRI data to compare the euglycemic and hypoglycemic conditions (see below, Dual regression procedure).

$f M R I$ data preprocessing. All fMRI time series were preprocessed using FMRIB (for Functional MRI of the Brain) FEAT (fMRI Expert Analysis Tool) version 5.98, part of the FMRIB Software Library (FSL; www. fmrib.ox.ac.uk/fsl; Smith et al., 2004; Beckmann et al., 2005) and included the following: (1) head motion correction using FLIRT (FMRIB Linear Registration Tool; Jenkinson et al., 2002); (2) non-brain removal using FSL BET (Brain Extraction Tool; Smith, 2002); (3) spatial smoothing by a Gaussian kernel (full-width at half-height, $5 \mathrm{~mm}$ ); (4) grandmean intensity normalization of the entire $4 \mathrm{D}$ dataset by a single multiplicative factor; and (5) high-pass temporal filtering by subtraction of a Gaussian-weighted least-squares straight line fitting with $\sigma=50 \mathrm{~s}$. Subjects' fMRI data were first registered to their high-resolution structural image using FLIRT (Jenkinson et al., 2002), which were then registered to MNI-152 standard space using FLIRT, followed by FNIRT (FMRIB Nonlinear Image Registration Tool; Jenkinson et al., 2012). All subjects' time series data were transformed into standard space at $2 \times$ $2 \times 2 \mathrm{~mm}$ resolution using the registration transformation matrices before the group ICA.

Group ICA. The entire fMRI time series for all subjects were concatenated in the temporal dimension and a group temporal concatenate probabilistic ICA (PICA; Beckmann et al., 2005) was implemented using FSL MELODIC (Multivariate Exploratory Linear Decomposition into Independent Components) version 3.09. The PICA estimates a set of spatiotemporal patterns that comprise the BOLD fMRI time series corresponding to underlying RSNs and artifactual processes that give rise to correlated BOLD signals. We set the number of components to 30 to obtain component spatial patterns consistent with the literature (Beckmann et al., 2005; Smith et al., 2009; Laird et al., 2011) for the most frequently reported major large-scale RSNs [e.g., default mode network $(\mathrm{DMN})$ ]. To ensure stable convergence of the ICA, it was run eight times and followed by a meta-level ICA using spatial maps from the eight decompositions (Smith et al., 2009) to identify the set of independent components (ICs), including RSNs and ICs related to artifacts, common to all participants. Through comparison of brain networks reported previously (Smith et al., 2009; Laird et al., 2011; Kullmann et al., 2012) and cross-correlation with template networks available at http://www.fmrib. ox.ac.uk/analysis/brainmap + rsns/ and the reference by Beckmann et al. (2005), the resulting ICs were inspected visually to identify RSNs involved in sensorimotor, cognitive, and limbic functions (Beckmann et al., 2005; Smith et al., 2009; Laird et al., 2011), interoception, RSNs investigated previously in a study of obesity by Kullmann et al. (2012), and an RSN containing the hypothalamus. This resulted in a total of seven components that were subjected to additional statistical analyses.

Dual regression procedure. To assess condition and group differences in RSN FC, we used the dual regression approach (Filippini et al., 2009), which has been shown to be a robust optimal approach for assessing intersubject variability with group ICA of fMRI data (Allen et al., 2012). This approach is informative of the specific shape of the RSN (i.e., localization and extent of all regions that constitute each RSN in a given subject or group of subjects), the degree of correlation among the time courses of the regions in the RSN (i.e., correlation coefficient), and the between network FC of large-scale RSNs, all together constituting a full picture of RSN behavior, which permits the assessment of group and condition differences.
To investigate differences in FC between the two conditions of euglycemia (EU) and hypoglycemia (HY), we defined condition-specific time courses by extracting, respectively, the first and last $10 \mathrm{~min}$ of the full 45 min time course during which blood glucose levels gradually declined. During the first $5 \mathrm{~min}$ of the full fMRI time course, blood glucose levels were clamped at euglycemic levels, after which glucose infusion was decreased to initiate onset hypoglycemia at a rate of $\sim 0.06$ $\mathrm{mmol} \cdot \mathrm{L}^{-1} \cdot \mathrm{min}^{-1}$. During the initial $10 \mathrm{~min}$ of the full $\mathrm{fMRI}$ time course (EU fMRI; Fig. 1), the time-weighted average \pm SE of blood glucose was $4.97 \pm 0.11 \mathrm{mmol} / \mathrm{L}$ for T1DM and $4.89 \pm 0.15 \mathrm{mmol} / \mathrm{L}$ for the control group. During the last $10 \mathrm{~min}$ of the full fMRI time course (HY fMRI; Fig. 1), the time-weighted average \pm SE of blood glucose was $3.36 \pm 0.08 \mathrm{mmol} / \mathrm{L}$ for T1DM and $3.27 \pm 0.12 \mathrm{mmol} / \mathrm{L}$ for the control group.

In the first stage of the dual regression, each subject's conditionspecific fMRI dataset (analyses were performed for both the EU and HY condition fMRI runs) was regressed against the full set of group IC maps identified from the full group ICA. This multiple spatial regression resulted in estimates of what are essentially the subject-specific time courses corresponding to each IC map. The full set of ICs was used at this stage, although final estimation of group/condition differences and statistical inference was done only for the seven components of interest. Including the full set of IC maps in the dual regression allowed for artifactual signals to also be modeled in the regression, thus reducing unexplained variance in the multiple regression. The subject-specific time courses from the first stage were used in a second multiple regression of each subjects' fMRI data against the full set of time courses. In this way, each subject's unique spatial pattern of the RSN identified in the group ICA was calculated.

Once all subject-specific spatial maps were calculated, estimation and inference of group/condition differences was done using a standard group general linear model (GLM) approach with nonparametric permutation testing with FSL Randomize (Nichols and Holmes, 2002; Smith et al., 2005). For example, to assess group differences between T1DM and controls during euglycemia, for each of the seven RSNs of interest, the set of subject-specific spatial maps (for the euglycemic condition) were collected into one file, resulting in seven sets of $263 \mathrm{D}$ spatial maps (one map for each of 16 patients with T1DM and 10 control subjects). Each set of spatial maps was assessed separately for group differences in within-network FC using nonparametric permutation testing with FSL Randomize (Nichols and Holmes, 2002; Smith et al., 2005), with cluster-based thresholding corrected for multiple comparisons by using the null distribution of the maximum cluster size (across the image). The cluster-forming threshold was $z=2.3$, and a familywise error corrected threshold of $p<0.05$ was used to define clusters showing significant differences in connectivity (Nichols and Hayasaka, 2003).

Differences in FC were assessed as follows: (1) between T1DM and control subjects during the euglycemic condition, using an unpaired $t$ test; (2) between T1DM and control subjects during the hypoglycemic condition, using an unpaired $t$ test; (3) between the euglycemic and hypoglycemic conditions for patients with T1DM and controls, separately, using a paired $t$ test; and (4) for differences between patients with T1DM and controls in the changes between hypoglycemia and euglycemia, using an unpaired $t$ test. In addition, HbAlc values and hypoglycemia symptom scores (mean centered within each group) were included as covariates in all models to determine whether or not these values explained any variability of the values around the means. For the models assessing group differences, mean centering within each group was used because $\mathrm{HbAlc}$ is used to establish the presence of T1DM, thus the covariates actually contribute to defining the two groups. Specifically, symptom scores during euglycemia were included in model 1 , symptom scores measured during hypoglycemia in model 2, and the change in symptom scores (hypoglycemia - euglycemia) in model 3. Reduced models were run to check for consistency in the estimation of effects (only results obtained with full models are presented as all reduced models were consistent with the full models). Corrections for comparisons across group/condition level tests were not done. This is consistent with 
A

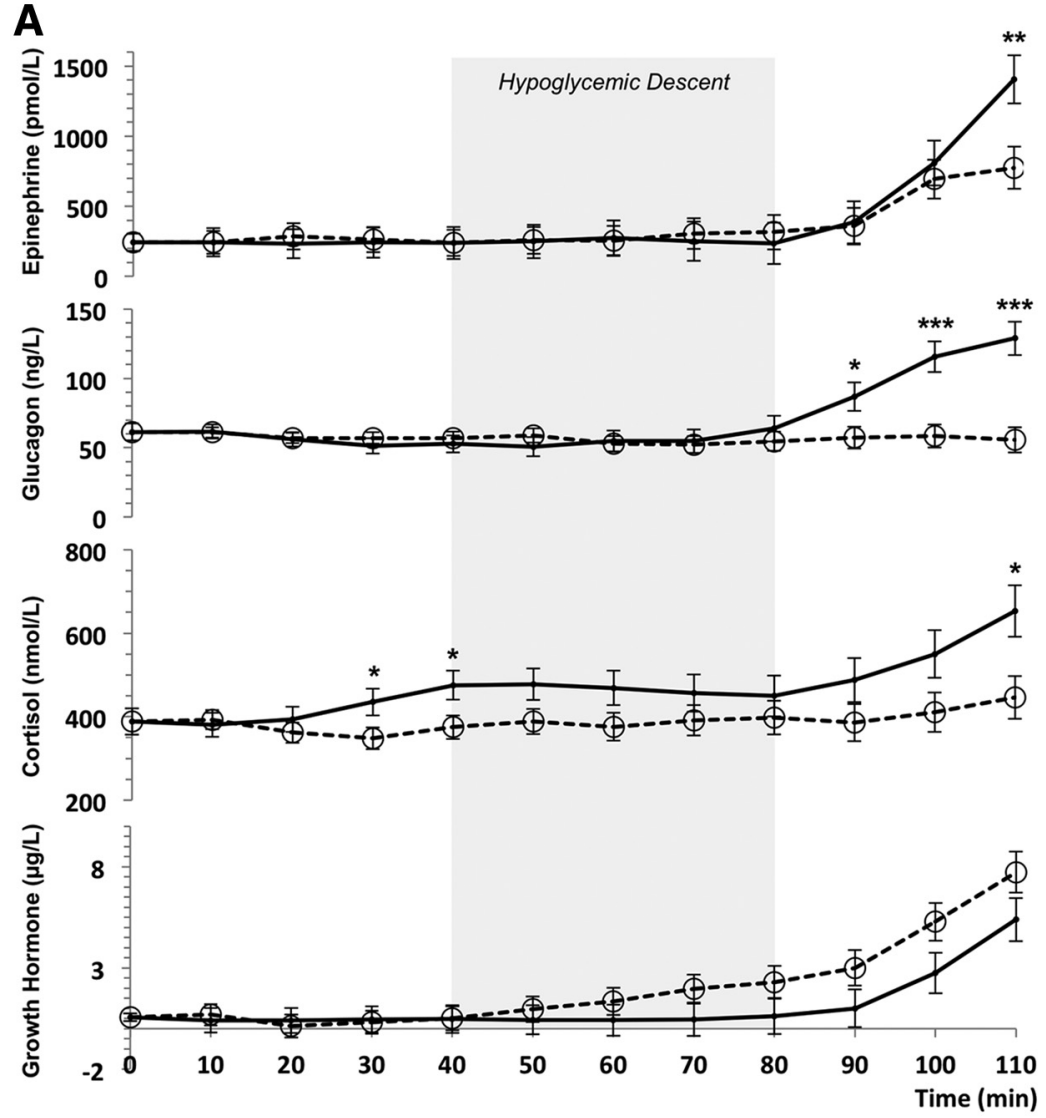

B
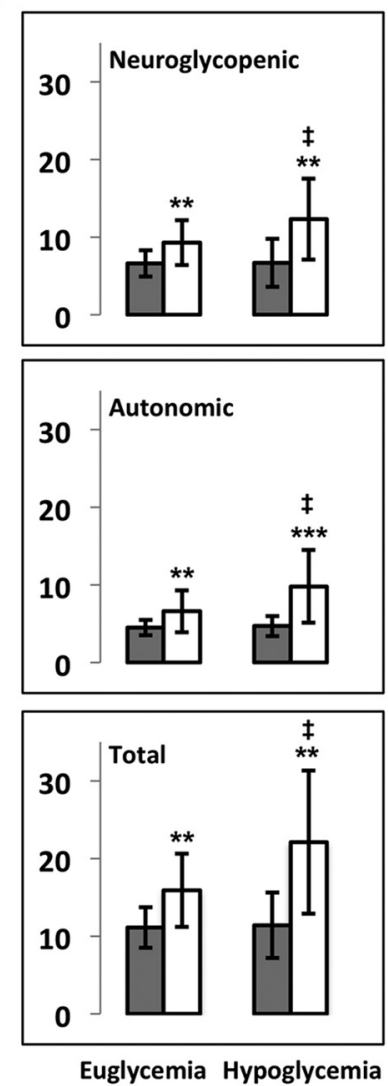

Figure 2. Counter-regulatory hormones and hypoglycemia symptom questionnaire scores. $\boldsymbol{A}$, Time courses of counter-regulatory hormones. Data are presented as mean \pm SE at each time point. The gray region indicates the time period of declining glycemia. Open circles and dashed line, T1DM; filled circles and solid line, nondiabetic controls (CONT). Comparison of T1DM versus CONT: * $p<$ $0.05,{ }^{* *} p<0.01,{ }^{* * *} p<0.001$. B , Hypoglycemia symptom questionnaire scores. Open bars, T1DM; filled bars, CONT. Data are presented as mean \pm SE. Top, Neuroglycopenic symptom score; middle, autonomic symptom score; bottom, total hypoglycemic (neuroglycopenic + autonomic) symptom score. Comparison of T1DM versus CONT: ${ }^{* *} p<0.01,{ }^{* *} p<0.001$. Comparison of euglycemia versus hypoglycemia: ${ }^{\ddagger} p<0.01$.

current practice in $\mathrm{fMRI}$ analyses to do a rigorous multiple comparisons correction for the $\sim 50,000$ voxelwise tests but not to further correct across the comparatively small number of tests at the group or condition level that are defined a priori in fMRI analyses. For example, conducting multiple hypothesis tests through different contrasts using a GLM is the most common style of analysis of fMRI data (and is what we have done in our analysis), yet these tests are not corrected for multiple contrasts (Perneger, 1998).

\section{Results}

\section{Demographics}

Demographic and clinical characteristics of the subjects are presented in Table 1 . As expected, the mean \pm SD HbA1c of patients with T1DM was significantly higher than that of nondiabetic controls ( $7.2 \pm 0.9$ vs $5.1 \pm 0.3 \% ; 55 \pm 7$ vs $34 \pm 2 \mathrm{mmol} / \mathrm{mol}$, $p<0.001)$, as was the average fasting plasma glucose level (6.3 \pm 1.6 vs $5.1 \pm 0.2 \mathrm{mmol} / \mathrm{L}, p<0.05)$. Average duration of diabetes for the patients was $16 \pm 6$ years. Historical information on patients' lifetime history of hypoglycemia included frequency of severe hypoglycemic reactions (range of $0-2$ episodes per patient), loss of consciousness (range of $0-2$ episodes per patient), seizures (range of $0-2$ episodes per patient), coma (no episodes in any patient), and emergency room visits as a result of hypoglycemia (range of $0-8$ episodes per patient). One patient with T1DM had reduced hypoglycemia awareness according to the Clarke questionnaire (Clarke et al., 1995). Groups were well matched for age $(p=0.97)$, gender $\left(\chi^{2}=0.88, p=0.35\right)$, fasting insulin $(p=0.38)$, and body mass index $(p=0.78)$. There were no significant differences between groups in either systolic ( $p=$ 0.50 ) or diastolic ( $p=0.52$ ) blood pressure assessed during the screening visit.

\section{Insulin clamp}

In this study, our goal was to investigate the changes in brain regional FC during the gradual induction of hypoglycemia to closely mimic the physiologic conditions experienced by an individual with T1DM during daily life. The onset of hypoglycemia in T1DM typically develops slowly over 30-60 min. Plasma glucose levels during the study are shown in Figure 1. Glucose levels were slightly, but significantly, higher in patients with T1DM versus controls during the basal period and the first $20 \mathrm{~min}$ of the euglycemic clamp (basal, $6.26 \pm 0.41 \mathrm{vs}$ $5.08 \pm 0.07 \mathrm{mmol} / \mathrm{L}, p<0.05)$. However, by the end of the euglycemic period and during the primary experimental period of declining glycemia, there were no significant differences in glucose levels between groups.

Levels of counter-regulatory hormones during the different phases of the study are presented in Figure $2 A$. Hormone levels did not rise appreciably in either group during the hypoglycemia phase because plasma glucose levels had just reached the typical threshold for a counter-regulatory response only a few minutes earlier. 
Table 2. Description of RSNs of interest and summary of $F$ C results

\begin{tabular}{lllll}
\hline RSN ID & RSN name & Regions in network & Functional associations from literature studies & FC results \\
\hline A & $\begin{array}{c}\text { Hypothalamus-basal } \\
\text { ganglia }\end{array}$ & $\begin{array}{c}\text { Hypothalamus, brainstem (ventral), basal ganglia } \\
\text { (caudate), temporal pole (uncus, hippocampus, }\end{array}$ & $\begin{array}{c}\text { Activation during hypoglycemia (Musen et al., } \\
\text { 2008) }\end{array}$ & $\begin{array}{c}\text { EU: T1DM }>\text { CONT: left putamen, left pallidum, } \\
\text { left inferior frontal gyrus, left superior frontal }\end{array}$ \\
& $\begin{array}{l}\text { parahippocampal gyrus, amygdala, superior } \\
\text { temporal gyrus), insula (posterior), thalamus }\end{array}$ & & gyrus (Fig. 3)
\end{tabular}

B Cerebellum-basal ganglia Cerebellum, basal ganglia (putamen and pallidum), dorsal brainstem, thalamus, anterior cingulate

C Temporal lobe

Temporal pole (anterior), hippocampus-parahippocampal gyrus, amygdala, insula, left orbitofrontal cortex, cerebellum

D DMN

E Sensorimotor cortex

F Medial prefrontal lobe

Posterior cingulate cortex/precuneus, frontal anterior cingulate/paracingulate cortex, inferior dorsomedial frontal cortex, superior lateral, occipital cortex, posterior parahippocampal cortex, thalamus

Precentral and postcentral gyri, Heschl's gyrus, central opercular cortex, thalamus

Anterior cingulate/paracingulate cortex, anterior frontal pole, orbital frontal cortex, insula (anterior)
Interoception, gustation, olfaction; emotional and CONT: $\mathrm{HY}<$ EU: right caudate, right anterior autonomic processing; autonomic responses, hunger, thirst, perception-somesthesia, pain (Smith et al., 2009; Laird et al., 2011; Page et al., 2011)

Interoceptive processing, hunger, olfactory and gustatory responses (Laird et al., 2011)

Active during rest, task negative (deactivation during tasks engaging cognition) (Smith et al., 2009)

Somatosensory and motor processing and planning (Smith et al., 2009; Laird et al., 2011)

Performance of cognitive tasks, executive control (Smith et al., 2009; Laird et al., 2011)

insula, right claustrum, right orbitofrontal cortex, right inferior frontal gyrus (Fig. 3).

CONT: Negative Correlation: $\Delta \mathrm{FC}_{\mathrm{HY}-\mathrm{EU}}$ and $\Delta \mathrm{SX}_{\mathrm{HY}-\mathrm{EU}}$, right putamen, hypothalamus (Fig. 5)

CONT: HY < EU: right anterior temporal fusiform cortex, right inferior temporal gyrus, right anterior insula, right hippocampus, right amygdala, right superior temporal gyrus (Fig. 3)

CONT: HY > EU: posterior cingulate cortex, precuneus (Fig. 3)

CONT: HY > EU: precentral and postcentral gyrus (Fig. 3)

T1DM: HY > EU: superior frontal gyrus, anterior cingulate cortex, right frontal pole, right precentral gyrus, right anterior insula (Fig. 3). T1DM: Correlation: $\triangle \mathrm{FC}_{\mathrm{HY}-\mathrm{EU}}$ and $\mathrm{HbA1c}$, medial prefrontal cortex, left anterior cingulate cortex, right insula, right basal ganglia (putamen) (Fig. 4)

Performance of cognitive tasks (Smith et al., 2009; No group or condition difference Laird et al., 2011)

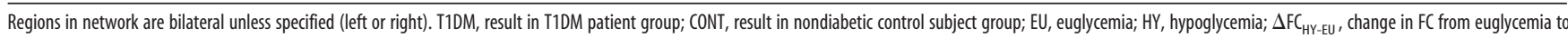
hypoglycemia; $\Delta \mathrm{SX}_{\mathrm{HY}-\mathrm{EU}}$, change in hypoglycemic symptom score from euglycemia to hypoglycemia.
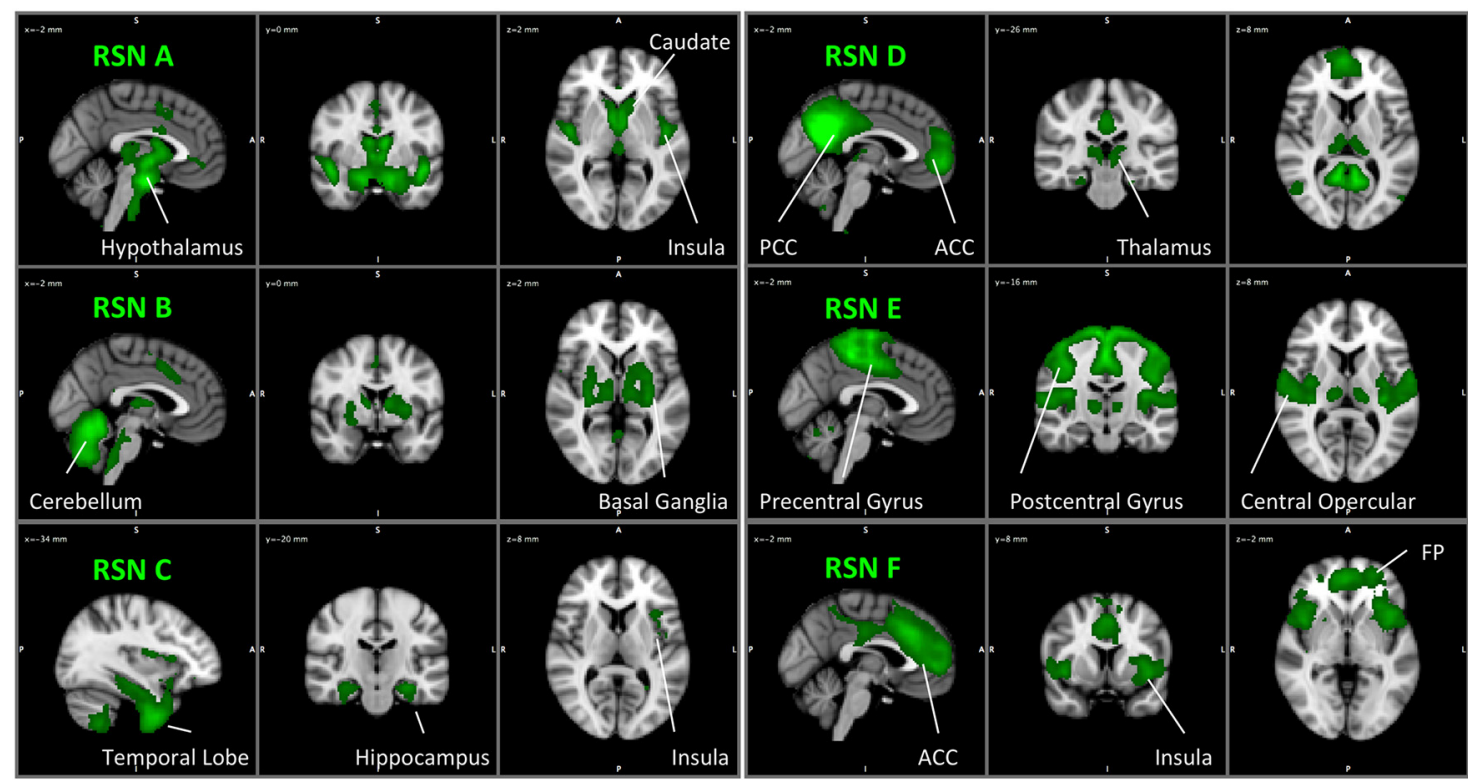

Figure 3. Spatial maps of RSNs of interest. Each RSN is shown in green overlaid onto orthographic views of the standard MNI-152 T1 brain image [ICBM152 nonlinear 6th generation asymmetric average brain stereotaxic registration brain model (Grabner et al., 2006)] shown in grayscale ( $2 \mathrm{~mm}$ isotropic resolution). Left, Sagittal view; middle, coronal view; right, axial view. Slice coordinates in standard MNI space are noted in the top left of each slice view. P, Posterior; A, anterior; R, right; L, left; ACC, anterior cingulate cortex; PCC, posterior cingulate cortex; FP, frontal pole. 


\section{Hypoglycemic symptom scores}

Scores from the questionnaire reporting hypoglycemic symptoms are summarized in Figure $2 B$. The scores from one patient with T1DM are missing from the analysis because the subject declined to perform the questionnaire. During both euglycemic and hypoglycemic conditions, patients with T1DM reported experiencing significantly stronger symptoms than controls (EU, 4.8 points higher, $p<0.003$; HY, 10.7 points higher, $p<0.002$ ). For the T1DM group, the average hypoglycemic symptom score increased significantly during hypoglycemia (change from EU to HY, $6.2 \pm 7.6$; Fig. 2); individual score changes ranged from -12 to 17 , indicating that some patients had reduced symptoms during hypoglycemia, whereas most others had increased symptoms (see Fig. 6). Although there was no significant change of average symptom score from euglycemia to hypoglycemia for the control group (change from EU to $\mathrm{HY}, 0.3 \pm 2.6$; Fig. 2), individual changes ranged from -4 to 6 , thus indicating that some controls had slightly reduced symptoms, most had no symptom change, whereas some others had moderately increased symptoms during hypoglycemia (see Fig. 6).

\section{Identification of RSNs from the whole-group ICA}

The functional significance of RSNs, which are found consistently across subjects, can be suggested by their spatial similarity with networks that have been shown in other studies to activate during given tasks (Damoiseaux et al., 2006). From the group ICA, we identified one RSN containing the hypothalamus that was similar to the hypothalamus seed-based network found in our previous report (Musen et al., 2008) and showed other functionally connected regions of ventral brainstem, bilateral caudate, and bilateral posterior insula. We additionally identified six RSNs showing a large overlap with those that have been implicated in feeding behaviors, insulin resistance, obesity, motor planning, and cognition (Smith et al., 2009; Laird et al., 2011; Kullmann et al., 2012). These RSNs are described in Table 2 with their previously reported functional significance. The spatial maps of the six RSNs that presented significant group or condition differences are shown in Figure 3. We labeled the RSNs with the letters A through G and gave them names (RSN A-RSN G) according to the main brain regions in the network or reported functional significance: (A) hypothalamus-basal ganglia (caudate); (B) cerebellum-basal ganglia (putamen, pallidum); (C) temporal lobe; (D) DMN; (E) sensorimotor cortex; (F) medial prefrontal lobe or executive control network (ECN); and (G) dorsolateral prefrontal lobe.

\section{Group and condition effects, and correlations of FC changes with symptoms and $\mathrm{HbA1c}$}

Results for FC group and condition comparisons are summarized in the last column of Table 2 and in Figure 4. In patients with T1DM, the left striatum and frontal lobe regions expressed higher FC with the hypothalamus network (RSN A) relative to controls during euglycemia. In controls, right anterior insula and ventral-frontal FC with the basal ganglia and cerebellum decreased (RSN B) and temporal lobe FC decreased (RSN C) during hypoglycemia relative to euglycemia; posterior cingulate and precuneus FC with DMN increased (RSN D) and sensorimotor cortex FC increased (RSN E). In patients with T1DM, right anterior insula and medial frontal FC increased during hypoglycemia relative to euglycemia (RSN F). There were no group or condition differences in the dorsolateral prefrontal lobe network (RSN G; data not shown).

Results for correlations between regional FC changes from euglycemia to hypoglycemia and the HbAlc of patients with
T1DM and the changes in symptom scores of nondiabetic controls are summarized in the last column of Table 2 and in Figures 5 and 6 . In patients with T1DM, the right insular cortex and medial frontal FC changes during hypoglycemia with the ECN (RSN F) were correlated positively with HbAlc levels $\left(R^{2}=\right.$ $0.733, p=0.001)$. In controls, the FC changes during hypoglycemia of the hypothalamus and basal ganglia with the cerebellumbasal ganglia network (RSN B) were correlated negatively with hypoglycemic symptom score changes $\left(R^{2}=0.831, p=0.003\right)$. A post hoc analysis revealed a stronger correlation of FC changes with neuroglycopenic symptom changes than with autonomic symptom changes (controls: neuroglycopenic, $R^{2}=0.749, p=$ 0.01 ; autonomic, $R^{2}=0.449, p=0.19$ ). No significant correlations with symptom changes were found for patients with T1DM (neuroglycopenic, $R^{2}=0.003, p=0.99$; autonomic, $R^{2}=0.007$, $p=0.98)$.

The data were reanalyzed excluding the patient with hypoglycemia unawareness, and there were no statistically significant or clinically meaningful differences in the results.

\section{Discussion}

We examined brain RSN FC of patients with T1DM compared with nondiabetic controls during experimentally induced mild hypoglycemia. We found both between- and within-group FC differences in subcortical and cortical brain regions involved in interoception, habitual feeding behavior, and cognition. We also found group-specific correlations between hypoglycemic FC, hypoglycemic symptoms, and HbAlc.

For the hypothalamus-basal ganglia (caudate) network (RSN A), patients with T1DM showed greater FC with left putamen than controls did during euglycemia. Patients also reported higher hypoglycemic symptom scores during euglycemia. The basal ganglia are involved in motor learning, motivation, and control of habitual behavior (Redgrave et al., 2010). The strength of FC in RSNs is influenced by experience (Albert et al., 2009; Lewis et al., 2009; Fornito and Bullmore, 2010; Corbetta, 2012). Thus, glycemic experience could have contributed to this connectivity alteration: repeated exposure to high or low glycemic levels could lead to neuroplastic adaptations that may have increased FC in this RSN. Such an adaptive mechanism could underlie a heightened ability to recognize or tendency to report hypoglycemia symptoms.

For the other RSNs, the two subject groups showed different patterns of changes during hypoglycemia. The control group showed decreased FC of the right anterior insula with the cerebellum and basal ganglia (putamen, pallidum) network (RSN B) and with the temporal pole network (RSN C). Furthermore, decreased FC of the right putamen, hypothalamus, and brainstem with RSN B was associated with an increase in hypoglycemic symptoms, more strongly with neuroglycopenic than autonomic ones. This suggests that the regional FC decreases with RSN B play a role in hypoglycemia awareness in controls. These RSNs have been associated with interoceptive, emotional, and autonomic processing; therefore, these changes in $\mathrm{FC}$ may provide a mechanism for processing information about glycemic-related physiologic changes. Changes in activation response to visual presentation of high-calorie foods have been observed in some of these regions (e.g., hypothalamus and putamen) during hypoglycemia in nondiabetic individuals, and these changes were associated with increased hunger ratings (Page et al., 2011). Thus, these FC changes may also play a role in motivation and food-seeking behavior. The right anterior insula supports visceral responses and interoceptive awareness (Craig, 2002; Critchley et al., 2004). 

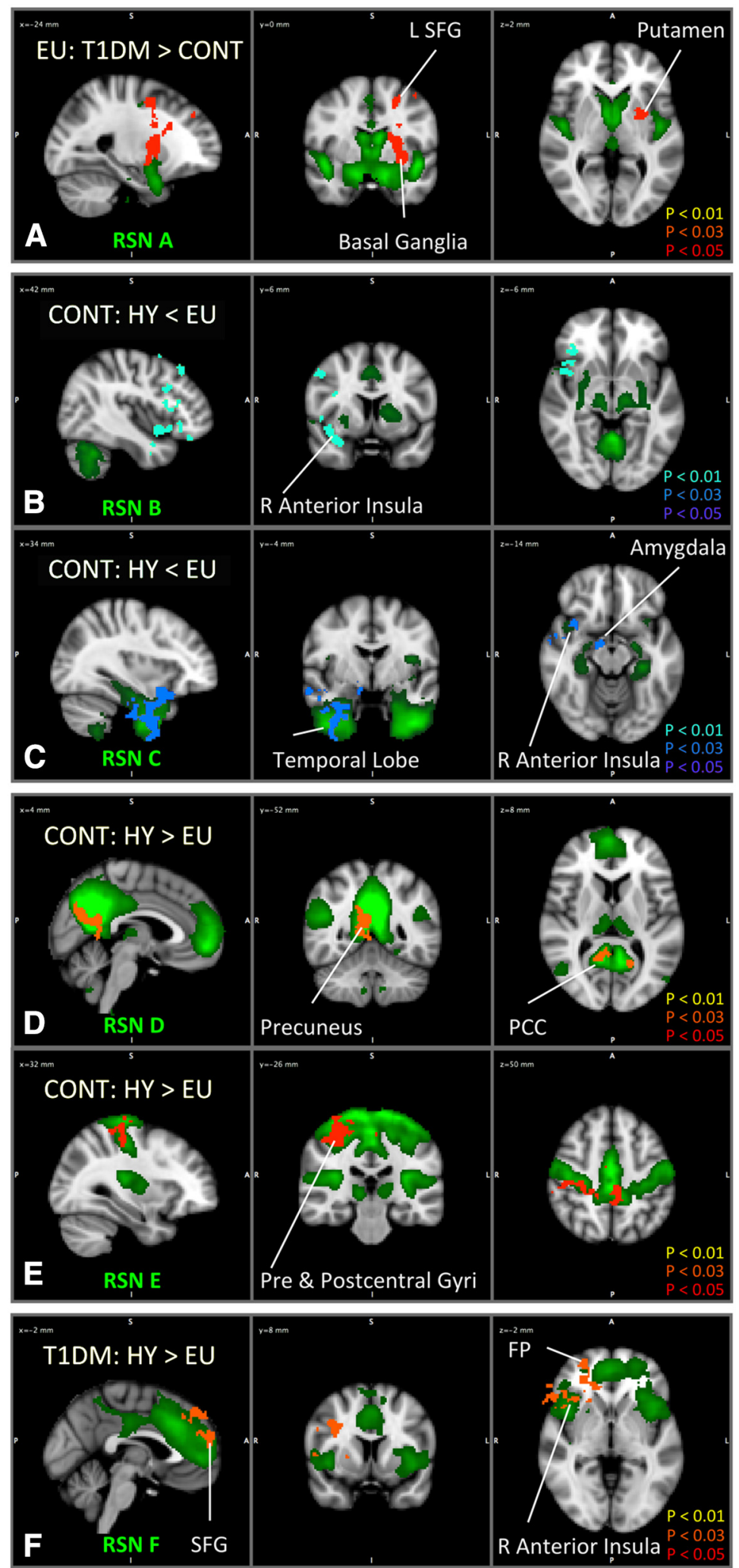

Figure 4. Group and condition effects. Regions of the RSNs that are common to both subject groups during the glycemic descent are shown in green. Regions showing significant group effects are shown in a color scale from red to yellow for higher FC in T1DM versus controls (red: $p<0.05$; orange: $p<0.03$; yellow: $p<0.01$ ), condition effects are shown in a color scale either from red to yellow for increased FC during hyperglycemia versus euglycemia (red, $p<0.05$; orange, $p<0.03$; yellow, $p<0.01$ ) or from dark blue to light blue for decreased FC during hyperglycemia versus euglycemia (dark blue, $p<0.05$; blue, $p<0.03$; light blue, $p<$ $0.01)$. Left, Sagittal view; middle, coronal view; right, axial view. Slice coordinates in standard MNI space are noted in the top left of each slice view. P, Posterior; A, anterior; R, right; L, left; SFG, superior frontal gyrus; PCC, posterior cingulate cortex; FP, frontal pole. $A$, EU: T1DM > CONT, Regions of higher within-network FC of the hypothalamus- basal ganglia RSN (RSN A) in patients with
Therefore, decreased FC of the right anterior insula with RSN B and RSN C during hypoglycemia could also reflect altered signaling of visceral changes contributing to the neuroglycopenic symptoms. Likewise, reduced FC of the right amygdala with RSN C could reflect compromised signaling of danger. The lack of these findings in T1DM suggests that repeated exposures to hypoglycemia may change FC, resulting in altered mediation of awareness or motivational signals.

The control group showed increased FC of the precuneus and posterior cingulate cortex in the DMN (RSN D) and increased FC of the precentral and postcentral cortical regions in the sensorimotor network (RSN E) during hypoglycemia. This is consistent with our previous observation of decreased deactivation in the posterior cingulate cortex during a working memory task in control subjects during hypoglycemia (Bolo et al., 2011). Increased connectivity of the DMN during hypoglycemia could interfere with task-related deactivations and activations. A study using positron emission tomography (PET) of ${ }^{15} \mathrm{O}$-labeled water to measure changes in brain perfusion in hypoglycemic-naive healthy controls during similarly mild early hypoglycemia (2.7 $\mathrm{mmol} / \mathrm{L}$; Teh et al., 2010) showed increased perfusion in the anterior cingulate cortex and thalamus, regions of the DMN, as well as in the striatum, ventromedial prefrontal cortex, and insula during later stable hyperglycemia. Our findings in the DMN could be related to the increases found by PET, with differences attributable to the methodological differences between our studies. Notably, Teh et al. (2010) compared euglycemia and early induction of hypoglycemia to later stable hypoglycemia and found more pronounced increases in more regions, whereas our study compared euglycemia and early hypoglycemia during a progressive glycemic descent. It is possible that we might have captured more changes of FC in similar regions if we had compared with resting state fMRI data acquired during a later stable hypoglycemic phase of the clamp. Together, the findings implicate in-

T1DM relative to controls during euglycemia. $B, C$, CONT:EU $>$ $H Y$, Regions of decreased within-network $\mathrm{FC}$ of the cerebellum-basal ganglia RSN (RSN B) and temporal lobe RSN (RSN C) during hypoglycemia relative to euglycemia in controls. $\boldsymbol{D}$, $E, C O N T$ : HY $>E$ EU, Regions of increased within-network FC of the default mode RSN (RSN D) and sensorimotor cortex RSN (RSN E) during hypoglycemia relative to euglycemia in controls. $\boldsymbol{F}, \mathrm{T} 1 \mathrm{DM}: \mathrm{HY}>\mathrm{EU}$, Regions of increased within-network FC of the medial prefrontal lobe RSN (RSN F) during hypoglycemia relative to euglycemia in patients with T1DM. 

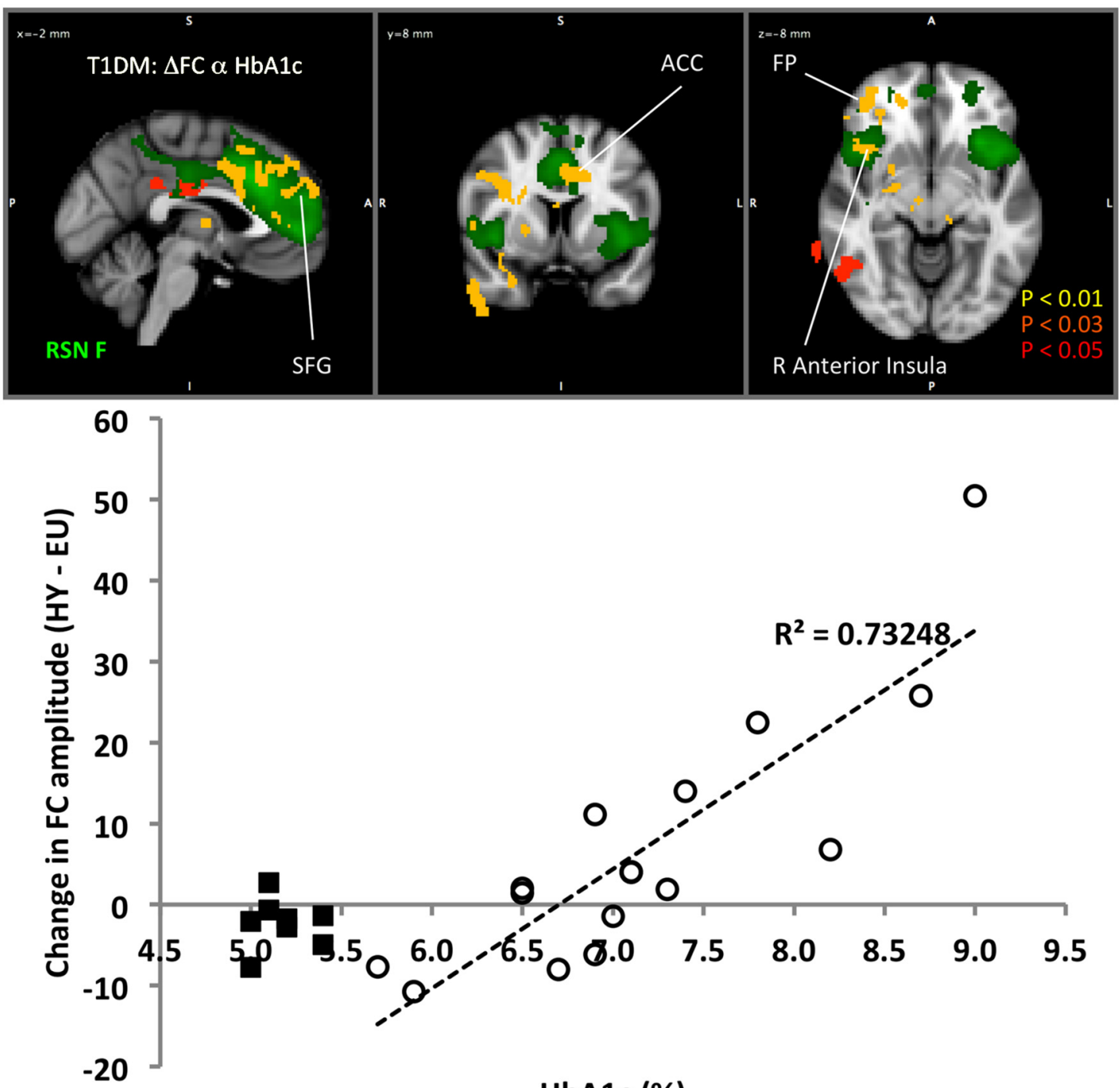

HbA1c (\%)

Figure 5. Correlation of $\mathrm{FC}$ changes with HbA1c. Top, T1DM: $\Delta \mathrm{FC} \alpha$ HbA1c, Regions of correlation $(\alpha)$ between HbA1c and within-network RSN F FC changes ( $\triangle \mathrm{FC})$ for T1DM. Regions of positive correlation with $\mathrm{HbA1}$ c are shown in red to yellow scale (red, $p<0.05$; orange, $p<0.03$; yellow, $p<0.01$ ). Regions of RSN $\mathrm{F}$ that are common to both subject groups during the glycemic descent are shown in green. Left, Sagittal view; middle, coronal view; right, axial view. Slice coordinates in standard MNI space are noted in the top left of each slice view. P, Posterior; A, anterior; R, right; L, left; SFG, superior frontal gyrus; ACC, anterior cingulate cortex; FP, frontal pole. Bottom, Plot of RSN F FC changes from euglycemia (EU) to hypoglycemia (HY) versus HbA1c. Open circles, T1DM; filled squares, nondiabetic controls (CONT); dashed line, linear fit for T1DM.

creased FC of regions of the DMN during induction of hypoglycemia in healthy hypoglycemic-naive control subjects. Our study suggests that this response is attenuated in T1DM. Alterations of sensorimotor RSN FC were also found in T1DM in a recent resting state fMRI study performed at basal glycemic levels (van Duinkerken et al., 2012): patients without microangiopathy showed increased (and patients with microangiopathy showed decreased) FC relative to controls. Although experimental conditions and patient groups were different, our findings of an attenuated sensorimotor network response to hypoglycemia in T1DM are compatible with those results and could be explained by similar early shifts or regional reorganizations of RSN FC related to chronic hyperglycemia.

Patients with T1DM showed increased FC during hypoglycemia of the right anterior insula, basal ganglia, and precentral frontal cortex with the medial prefrontal cortex network (RSN F), also known as the ECN, which plays an important role in cognition. This increased prefrontal connectivity is also compatible with our previous finding of increased prefrontal activation dur- ing working memory (Bolo et al., 2011). Increased FC in this cognitive processing-related network may be an adaptation to multiple episodes of hypoglycemia reflecting increased maintenance of cognitive function during hypoglycemic challenges. Similar medial prefrontal cortex regions were found to have increased FC in obese versus lean individuals during visual processing of food stimuli (Kullmann et al., 2013). Thus, these changes could also be related to food-seeking behavior and to the tendency for individuals with T1DM to gain weight.

Changes in brainstem and right putamen connectivity with the cerebellum-basal ganglia network (RSN B) during hypoglycemia were correlated with changes in self-reported hypoglycemic symptom scores for controls only. This suggests a role for these regions in hypoglycemia awareness, which could modulate motivation for food-seeking behaviors. The correlation was dominated by neuroglycopenic symptoms. For controls, the experience of hypoglycemia is novel. Because the basal ganglia are involved in rule learning and habit control, these connectivity changes may be part of a learning 

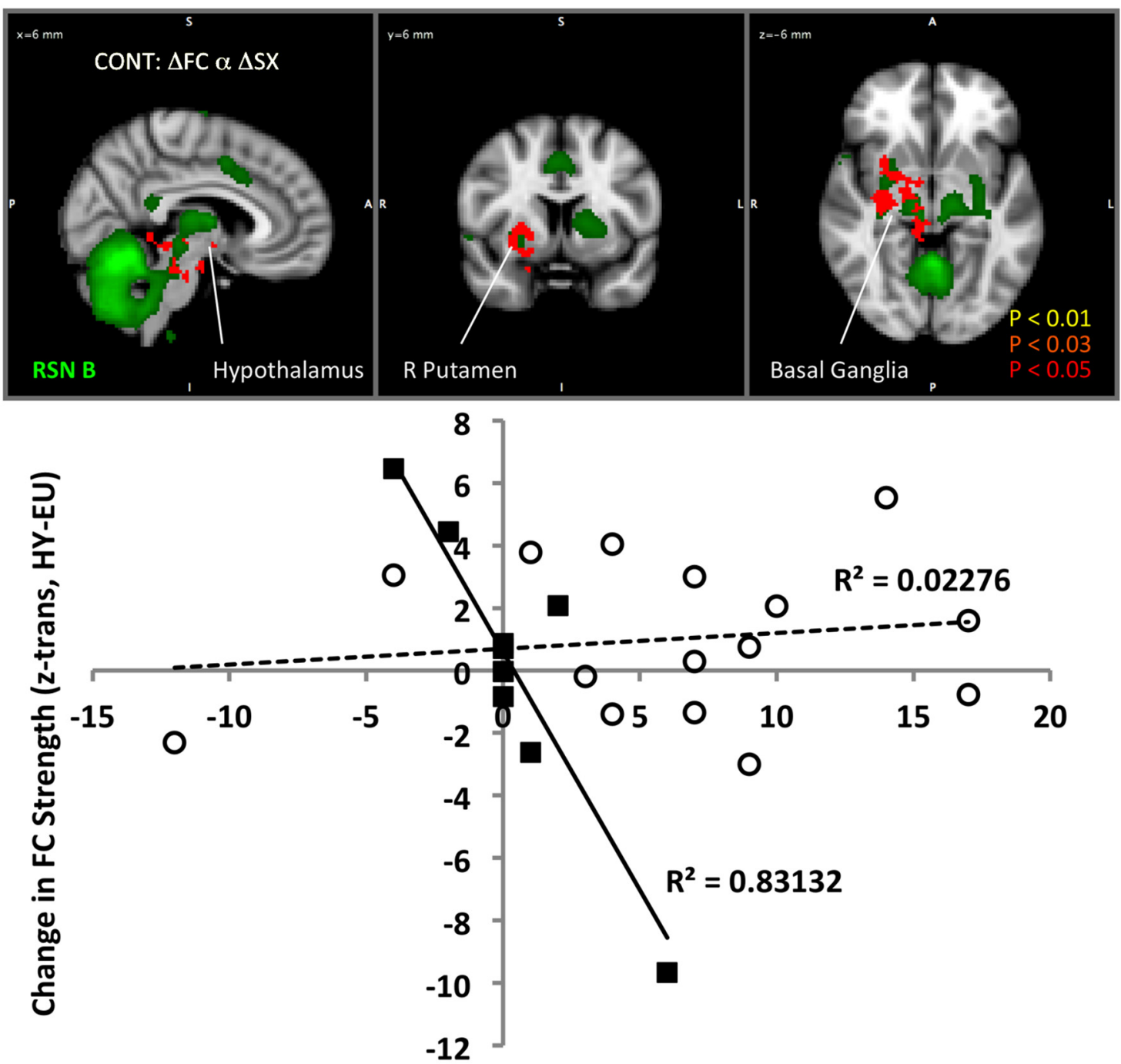

\section{Change in Hypoglycemic Symptom Score (HY-EU)}

Figure 6. Correlation of $\mathrm{FC}$ changes with symptom score changes. Top, CONT: $\triangle \mathrm{FC} \alpha \Delta \mathrm{SX}$, Regions of stronger correlation $(\alpha)$ between symptom score changes ( $\triangle \mathrm{SX}$ ) and within-network RSN $B F C$ changes $(\triangle \mathrm{FC}$ ) in controls relative to T1DM (CONT $>$ T1DM). Regions of stronger correlation are shown in red to yellow scale (red, $p<0.05$; orange, $p<0.03$; yellow, $p<0.01)$. Regions of RSN B that are common to both subject groups during the glycemic decline are shown in green. P, Posterior; A, anterior; R, right; L, left. Bottom, Plot of RSN B FC changes versus hypoglycemic symptom score changes from euglycemia (EU) to hypoglycemia (HY). The z transforms of FC change (HY - EU) of cerebellum-basal ganglia RSN within-network FC, averaged over the voxels of the right basal ganglia with the strongest between-group difference $(p<0.05)$, are plotted on the vertical axis for each individual. Open circles, T1DM; filled squares, nondiabetic controls (CONT); dashed line, linear fit for T1DM; solid line, linear fit for controls.

process associating neuroglycopenic perceptions with low blood glucose. Such a transitory learning process could explain the lack of correlation in patients with T1DM who have experienced several hypoglycemic episodes.

Changes in ECN FC in T1DM during hypoglycemia were correlated with $\mathrm{HbA1c}$, suggesting that more frequent exposure to higher glycemic levels and consequently larger excursions during hypoglycemic episodes may have altered regional glucose and neurotransmitter metabolism in this network with a resulting effect on FC. The association of connectivity changes with HbAlc implies an association with previous glycemic control: insula and prefrontal hypoglycemic FC was decreased for patients with HbAlc closer to the normal range (approximately $<7.0 \%, 53$ $\mathrm{mmol} / \mathrm{mol}$ ) and increased for patients with higher HbAlc (approximately $\geq 7.0 \%, 53 \mathrm{mmol} / \mathrm{mol}$ ). Thus, connectivity changes in T1DM with poor glycemic control may reflect a metabolic adaptation to severe chronic hyperglycemia. The FC changes for patients with T1DM with low HbAlc were in the same range as those for nondiabetic controls. Studies using proton magnetic resonance spectroscopy have shown that patients with T1DM maintain brain glutamate levels during moderate hypoglycemia compared with controls who show significant decreases (Bischof et al., 2006; Terpstra et al., 2014). Thus, the hyperconnectivity of the ECN during hypoglycemia could be attributable to the maintenance of high glutamatergic neurotransmission or glutamate levels driven by increased glucose or energetic metabolism related to persistent hyperglycemia.

Furthermore, we note that the regions of the ECN showing increased connectivity during hypoglycemia are similar to the superior prefrontal cortical regions in which we have also recently reported cortical thickness deficits in T1DM potentially related to glutamate hyperactivity and its excitotoxic effects (Lyoo et al., 2012) and associated with cognitive deficits and depression. Together, our findings suggest that the ECN may also 
present prefrontal hyperconnectivity during persistent hyperglycemia in T1DM, which may mediate cognitive and emotional dysfunction. We are currently investigating this hypothesis.

Plasma glucose levels were slightly but significantly higher in patients with T1DM versus controls $\sim 1 \mathrm{~h}$ before the start of the study but were brought to equivalent levels during the euglycemic clamp. It is possible that differences between patients and controls in the rate of glucose washout from brain tissue may have affected our results.

Changes in FC and hypoglycemic symptoms occurred without significant changes in counter-regulatory hormones. During declining glycemia, plasma glucose was only below the level required for activation of a hypoglycemic response $(\sim 3.9 \mathrm{mmol} / \mathrm{L})$ for the final 10-15 min. This suggests that symptomatic responses and changes in connectivity occurred as a result of low plasma glucose levels and were not mediated by an increase in counter-regulatory hormones.

The overall power of the comparisons in our study may be limited because of the relatively small number of participants in each group ( $n=10$ for controls and $n=16$ for T1DM). Also, the unequal number of subjects in each group and the use of unpaired and paired $t$ test, respectively, in the group and condition comparisons results in unequal power between the comparisons. Additional studies with larger groups are needed.

In summary, our study suggests that changes in FC within RSNs that contain core nodes including hypothalamus, basal ganglia, insula, default mode, sensorimotor and cingulate cortices, and cerebellum play an important role in awareness and behavioral response to hypoglycemia. Hypoglycemia induced changes in multiple RSNs in controls and in the ECN in T1DM. The different FC response between groups may stem from familiarity with hypoglycemia in T1DM. Repeated exposure to high and low glycemic levels in T1DM may reinforce FC via neuroplasticity-related mechanisms and shift a multiple network response to a frontal ECN response. This could reflect a cognitive neuroplastic adaptation to chronic hyperglycemic levels, which may normalize with good glycemic control. The frequent exposure to high and low blood glucose levels also likely perturbs brain glucose, energetic, and neurotransmitter metabolic pathways by metabolic adaptive mechanisms (Öz et al., 2012; Seaquist and Öz, 2013; van de Ven et al., 2013; Terpstra et al., 2014), which could underlie broad perturbations of RSN FC patterns. More studies linking brain metabolism and function are needed to understand the mechanisms by which glucose metabolic dysregulation may affect cognition, emotion, and behavior.

\section{References}

Albert NB, Robertson EM, Miall RC (2009) The resting human brain and motor learning. Curr Biol 19:1023-1027. CrossRef Medline

Allen EA, Erhardt EB, Wei Y, Eichele T, Calhoun VD (2012) Capturing inter-subject variability with group independent component analysis of fMRI data: a simulation study. Neuroimage 59:4141-4159. CrossRef Medline

Beckmann CF, DeLuca M, Devlin JT, Smith SM (2005) Investigations into resting-state connectivity using independent component analysis. Philos Trans R Soc Lond B Biol Sci 360:1001-1013. CrossRef Medline

Bingham EM, Hopkins D, Smith D, Pernet A, Hallett W, Reed L, Marsden PK, Amiel SA (2002) The role of insulin in human brain glucose metabolism: an 18fluoro-deoxyglucose positron emission tomography study. Diabetes 51:3384-3390. CrossRef Medline

Bischof MG, Brehm A, Bernroider E, Krssák M, Mlynárik V, Krebs M, Roden M (2006) Cerebral glutamate metabolism during hypoglycaemia in healthy and type 1 diabetic humans. Eur J Clin Invest 36:164-169. CrossRef Medline

Bolo NR, Musen G, Jacobson AM, Weinger K, McCartney RL, Flores V,
Renshaw PF, Simonson DC (2011) Brain activation during working memory is altered in patients with type 1 diabetes during hypoglycemia. Diabetes 60:3256-3264. CrossRef Medline

Clarke WL, Cox DJ, Gonder-Frederick LA, Julian D, Schlundt D, Polonsky W (1995) Reduced awareness of hypoglycemia in adults with IDDM. A prospective study of hypoglycemic frequency and associated symptoms. Diabetes Care 18:517-522. CrossRef Medline

Corbetta M (2012) Functional connectivity and neurological recovery. Dev Psychobiol 54:239-253. CrossRef Medline

Craft S, Watson GS (2004) Insulin and neurodegenerative disease: shared and specific mechanisms. Lancet Neurol 3:169-178. CrossRef Medline

Craig AD (2002) How do you feel? Interoception: the sense of the physiological condition of the body. Nat Rev Neurosci 3:655-666. CrossRef Medline

Critchley HD, Wiens S, Rotshtein P, Ohman A, Dolan RJ (2004) Neural systems supporting interoceptive awareness. Nat Neurosci 7:189-195. CrossRef Medline

Damoiseaux JS, Rombouts SA, Barkhof F, Scheltens P, Stam CJ, Smith SM, Beckmann CF (2006) Consistent resting-state networks across healthy subjects. Proc Natl Acad Sci U S A 103:13848-13853. CrossRef Medline

Filippini N, MacIntosh BJ, Hough MG, Goodwin GM, Frisoni GB, Smith SM, Matthews PM, Beckmann CF, Mackay CE (2009) Distinct patterns of brain activity in young carriers of the APOE-epsilon4 allele. Proc Natl Acad Sci U S A 106:7209-7214. CrossRef Medline

Fornito A, Bullmore ET (2010) What can spontaneous fluctuations of the blood oxygenation-level-dependent signal tell us about psychiatric disorders? Curr Opin Psychiatry 23:239-249. CrossRef Medline

Fox MD, Raichle ME (2007) Spontaneous fluctuations in brain activity observed with functional magnetic resonance imaging. Nat Rev Neurosci 8:700-711. CrossRef Medline

Grabner G, Janke AL, Budge MM, Smith D, Pruessner J, Collins DL (2006) Symmetric atlasing and model based segmentation: an application to the hippocampus in older adults. Med Image Comput Comput Assist Interv 9:58-66. Medline

Jenkinson M, Bannister P, Brady M, Smith S (2002) Improved optimization for the robust and accurate linear registration and motion correction of brain images. Neuroimage 17:825-841. CrossRef Medline

Jenkinson M, Beckmann CF, Behrens TE, Woolrich MW, Smith SM (2012) FSL. Neuroimage 62:782-790. CrossRef Medline

Kapogiannis D, Reiter DA, Willette AA, Mattson MP (2013) Posteromedial cortex glutamate and GABA predict intrinsic functional connectivity of the default mode network. Neuroimage 64:112-119. CrossRef Medline

Kullmann S, Heni M, Veit R, Ketterer C, Schick F, Häring HU, Fritsche A, Preissl H (2012) The obese brain: association of body mass index and insulin sensitivity with resting state network functional connectivity. Hum Brain Mapp 33:1052-1061. CrossRef Medline

Kullmann S, Pape AA, Heni M, Ketterer C, Schick F, Häring HU, Fritsche A, Preissl H, Veit R (2013) Functional network connectivity underlying food processing: disturbed salience and visual processing in overweight and obese adults. Cereb Cortex 23:1247-1256. CrossRef Medline

Laird AR, Fox PM, Eickhoff SB, Turner JA, Ray KL, McKay DR, Glahn DC, Beckmann CF, Smith SM, Fox PT (2011) Behavioral interpretations of intrinsic connectivity networks. J Cogn Neurosci 23:4022-4037. CrossRef Medline

Lewis CM, Baldassarre A, Committeri G, Romani GL, Corbetta M (2009) Learning sculpts the spontaneous activity of the resting human brain. Proc Natl Acad Sci U S A 106:17558-17563. CrossRef Medline

Lyoo IK, Yoon SJ, Musen G, Simonson DC, Weinger K, Bolo N, Ryan CM, Kim JE, Renshaw PF, Jacobson AM (2009) Altered prefrontal glutamateglutamine-gamma-aminobutyric acid levels and relation to low cognitive performance and depressive symptoms in type 1 diabetes mellitus. Arch Gen Psychiatry 66:878-887. CrossRef Medline

Lyoo IK, Yoon S, Jacobson AM, Hwang J, Musen G, Kim JE, Simonson DC, Bae S, Bolo N, Kim DJ, Weinger K, Lee JH, Ryan CM, Renshaw PF (2012) Prefrontal cortical deficits in type 1 diabetes mellitus: brain correlates of comorbid depression. Arch Gen Psychiatry 69:1267-1276. CrossRef Medline

Musen G, Simonson DC, Bolo NR, Driscoll A, Weinger K, Raji A, Théberge J, Renshaw PF, Jacobson AM (2008) Regional brain activation during hypoglycemia in type 1 diabetes. J Clin Endocrinol Metab 93:1450-1457. CrossRef Medline

Nichols T, Hayasaka S (2003) Controlling the familywise error rate in func- 
tional neuroimaging: a comparative review. Stat Methods Med Res 12: 419-446. CrossRef Medline

Nichols TE, Holmes AP (2002) Nonparametric permutation tests for functional neuroimaging: a primer with examples. Hum Brain Mapp 15:1-25. CrossRef Medline

Öz G, Tesfaye N, Kumar A, Deelchand DK, Eberly LE, Seaquist ER (2012) Brain glycogen content and metabolism in subjects with type 1 diabetes and hypoglycemia unawareness. J Cereb Blood Flow Metab 32:256-263. CrossRef Medline

Page KA, Seo D, Belfort-DeAguiar R, Lacadie C, Dzuira J, Naik S, Amarnath S, Constable RT, Sherwin RS, Sinha R (2011) Circulating glucose levels modulate neural control of desire for high-calorie foods in humans. J Clin Invest 121:4161-4169. CrossRef Medline

Perneger TV (1998) What's wrong with Bonferroni adjustments. BMJ 316: 1236-1238. CrossRef Medline

Redgrave P, Rodriguez M, Smith Y, Rodriguez-Oroz MC, Lehericy S, Bergman H, Agid Y, DeLong MR, Obeso JA (2010) Goal-directed and habitual control in the basal ganglia: implications for Parkinson's disease. Nat Rev Neurosci 11:760-772. CrossRef Medline

Schulingkamp RJ, Pagano TC, Hung D, Raffa RB (2000) Insulin receptors and insulin action in the brain: review and clinical implications. Neurosci Biobehav Rev 24:855-872. CrossRef Medline

Seaquist ER, Öz G (2013) Diabetes: does lactate sustain brain metabolism during hypoglycaemia? Nat Rev Endocrinol 9:386-387. CrossRef Medline

Simmons WK, Avery JA, Barcalow JC, Bodurka J, Drevets WC, Bellgowan P (2013) Keeping the body in mind: insula functional organization and functional connectivity integrate interoceptive, exteroceptive, and emotional awareness. Hum Brain Mapp 34:2944-2958. CrossRef Medline

Smith SM (2002) Fast robust automated brain extraction. Hum Brain Mapp 17:143-155. CrossRef Medline

Smith SM, Jenkinson M, Woolrich MW, Beckmann CF, Behrens TEJ,
Johansen-Berg H, Bannister PR, De Luca M, Drobnjak I, Flitney DE, Niazy RK, Saunders J, Vickers J, Zhang Y, De Stefano N, Brady JM, Matthews PM (2004) Advances in functional and structural MR image analysis and implementation as FSL. Neuroimage 23 [Suppl 1]:S208-S219. CrossRef

Smith SM, Beckmann CF, Ramnani N, Woolrich MW, Bannister PR, Jenkinson M, Matthews PM, McGonigle DJ (2005) Variability in fMRI: A reexamination of inter-session differences. Hum Brain Mapp 24:248-257. CrossRef Medline

Smith SM, Fox PT, Miller KL, Glahn DC, Fox PM, Mackay CE, Filippini N, Watkins KE, Toro R, Laird AR, Beckmann CF (2009) Correspondence of the brain's functional architecture during activation and rest. Proc Natl Acad Sci U S A 106:13040-13045. CrossRef Medline

Teh MM, Dunn JT, Choudhary P, Samarasinghe Y, Macdonald I, O'Doherty M, Marsden P, Reed LJ, Amiel SA (2010) Evolution and resolution of human brain perfusion responses to the stress of induced hypoglycemia. Neuroimage 53:584-592. CrossRef Medline

Terpstra M, Moheet A, Kumar A, Eberly LE, Seaquist E, Öz G (2014) Changes in human brain glutamate concentration during hypoglycemia: insights into cerebral adaptations in hypoglycemia-associated autonomic failure in type 1 diabetes. J Cereb Blood Flow Metab 34:876-882. CrossRef Medline

van de Ven K, Tack CJ, Heerschap A, van der Graaf M, de Galan BE (2013) Patients with type 1 diabetes exhibit altered cerebral metabolism during hypoglycemia. J Clin Invest 123:623-629. CrossRef Medline

van Duinkerken E, Schoonheim MM, Sanz-Arigita EJ, Ijzerman RG, Moll AC, Snoek FJ, Ryan CM, Klein M, Diamant M, Barkhof F (2012) Restingstate brain networks in type 1 diabetic patients with and without microangiopathy and their relation to cognitive functions and disease variables. Diabetes 61:1814-1821. CrossRef Medline

Yin HH, Knowlton BJ (2006) The role of the basal ganglia in habit formation. Nat Rev Neurosci 7:464-476. CrossRef Medline 